\title{
Changes in Quality of Life in Older Hemodialysis Patients: A Cohort Study on Dialysis Outcomes and Practice Patterns
}

\author{
Ayumi Ishiwataria Shungo Yamamoto $^{\text {b, c }}$ Shingo Fukumad \\ Takeshi Hasegawa $^{\mathrm{e}-\mathrm{h}}$ Sachiko Wakai ${ }^{\mathrm{a}}$ Masaomi Nangaku ${ }^{\mathrm{i}}$ \\ aDivision of Nephrology, Okubo Hospital, Tokyo, Japan; ${ }^{b}$ Department of Healthcare Epidemiology, School of \\ Public Health in the Graduate School of Medicine, Kyoto University, Kyoto, Japan; 'Institute for Health Outcomes

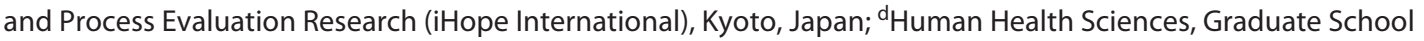 \\ of Medicine, Kyoto University, Kyoto, Japan; eShowa University Research Administration Center (SURAC), Showa \\ University, Tokyo, Japan; fDepartment of Hygiene, Public Health, and Preventive Medicine, Graduate School of \\ Medicine, Showa University, Tokyo, Japan; 9Division of Nephrology, Department of Medicine, School of Medicine, \\ Showa University, Tokyo, Japan; ${ }^{h}$ Center for Innovative Research for Communities and Clinical Excellence, \\ Fukushima Medical University, Fukushima, Japan; 'Division of Nephrology and Endocrinology, The University of \\ Tokyo Graduate School of Medicine, Tokyo, Japan
}

\section{Keywords}

Dialysis Outcomes and Practice Patterns Study ·

Hemodialysis · Quality of life

\begin{abstract}
Background: Despite improvements in dialysis treatment, mortality rates remain high, especially among older hemodialysis patients. Quality of life (QOL) among hemodialysis patients is strongly associated with higher risk of death. This study aimed to describe the health-related QOL and its change in older maintenance hemodialysis patients and to demonstrate characteristics associated with health-related QOL. Methods: Data on 892 maintenance hemodialysis patients aged 60 years or older who were surveyed using the Kidney Disease Quality of Life Short Form at baseline and 2 years after study enrollment in phases 4 (2009-2011) and 5 (2012-2014) of the Japanese Dialysis Outcomes and Practice Patterns Study were analyzed. We categorized participants into 3 age groups (60-69, 70-79, and $\geq 80$ years) and de-
\end{abstract}

\begin{tabular}{ll}
\hline KARGER & $\begin{array}{l}\text { (c) } 2020 \text { The Author(s) } \\
\text { Published by S. Karger AG, Basel }\end{array}$ \\
karger@karger.com & This is an Open Access article licensed under the Creative Commons \\
www.karger.com/ajn & $\begin{array}{l}\text { Attribution-NonCommercial-4.0 International License (CC BY-NC) } \\
\text { (http://www.karger.com/Services/OpenAccessicense), applicable to } \\
\text { the online version of the article only. Usage and distribution for com- } \\
\text { mercial purposes requires written permission. }\end{array}$
\end{tabular}

scribed baseline physical component summary (PCS) and mental component summary (MCS) scores, as well as their distribution of changes after 2 years across each category. Results: Hemodialysis patients aged $70-79$ years and $\geq 80$ years had lower PCS scores than those aged 60-69 years (median: $70-79$ years $=43.1$; interquartile range [IQR], 35.2$49.4 ; \geq 80$ years $=38.8 ; \mid \mathrm{QR}, 31.6-43.8 ; 60-69$ years $=45.4 ; \mathrm{IQR}$, $37.5-51.4 ; p<0.001)$. In contrast, MCS scores did not significantly differ by age category (70-79 years $=45.6$; IQR, 38.4$53.7 ; \geq 80$ years $=45.4 ; \mathrm{IQR}, 36.9-55.1 ; 60-69$ years $=46.8 ; \mathrm{IQR}$, 39.5-55.7; $p=0.1$ ). As dialysis vintage lengthened, the PCS score significantly became lower, whereas no association was found with change in the MCS score. The MCS score declined over time in older patients, especially among those aged 80 years and older after 2 years' follow-up. Conclusions: Physical QOL became worse as dialysis vintage lengthened. In contrast, mental QOL declined over time within a relatively short period among older maintenance hemodialysis patients.

(c) 2020 The Author(s)

Published by S. Karger AG, Basel
Ayumi Ishiwatari

Division of Nephrology, Okubo Hospital

2-44-1 Kabuki-cho, Shinjuku-ku

Tokyo 160-8488 (Japan)

ayumi_ishiwatari@tokyo-hmt.jp 


\section{Introduction}

Japan has the highest percentage of elderly people in the world, and the number of patients with chronic kidney disease is rising markedly. The aging of the population, together with higher rates of diabetes, hypertension, and cardiovascular disease, has caused the incidence of end-stage renal disease (ESRD) and demand for dialysis in older patients to also increase, both in Japan and worldwide [1]. Despite improvements in dialysis treatments, mortality rates remain high, especially for older patients [2]. Furthermore, mortality risk soon after initiation of hemodialysis is also high worldwide [3]. The functional disability of the condition is more closely related with early death in the elderly than younger patients [4]. However, it is unknown whether the self-reported, subjective quality of life (QOL) of older hemodialysis patients changes over time.

Health-related quality of life (HR-QOL) in hemodialysis patients is dramatically lower than that in the general population and strongly associated with higher risk of death and hospitalization, independently of a range of demographic and comorbid factors [5]. The Kidney Disease Outcomes Quality Initiative (KDOQI) guidelines emphasize the importance of HR-QOL as a key outcome and recommend repeated ongoing assessment of HR-QOL measures to serve as a parameter in monitoring the quality of care delivered to maintenance dialysis patients [6]. A previous study described changes in HR-QOL among patients on hemodialysis and showed that the most recent HR-QOL score was associated with mortality [7].

Since HR-QOL is a measure of the well-being of hemodialysis patients and an independent prognostic predictor [8], understanding the natural history of QOL and its change among older maintenance hemodialysis patients is important. To date, however, no study has described changes in HR-QOL with a focus on older patients on hemodialysis. In this study, our primary aim was to describe the Kidney Disease Quality of Life Short Form (KDQOL-SF) physical and mental score and its changes in older hemodialysis patients and to demonstrate demographic and clinical factors associated with HR-QOL.

\section{Materials and Methods}

\section{Design, Setting, and Participants}

We used data from phases 4 (2009-2011) and 5 (2012-2014) of the Japanese Dialysis Outcomes and Practice Patterns Study (JDOPPS), which is part of the DOPPS, an international prospective cohort study of in-center hemodialysis patients. The study design, data elements, and methodology of the DOPPS have been detailed elsewhere [9]. In the present study, eligible participants were maintenance hemodialysis patients aged 60 years or older who were surveyed using the KDQOL-SF at baseline and 2 years after study enrollment. Laboratory data were obtained early in the week (Monday or Tuesday).

\section{Outcomes}

The primary outcomes of interest were the change in KDQOLSF physical component summary (PCS) and mental component summary (MCS) scores in 2 years. DOPPS participants completed self-administered questionnaires, including KDQOL-SF, typically within 2 months of study entry, and then yearly.

\section{Statistical Analysis}

We categorized participants into 3 age groups (60-69, 70-79, and $\geq 80$ years) according to their age at entry into the present study. We summarized baseline participants characteristics and hemodialysis treatment characteristics based on the categories of age groups as a percentage for categorical variables and median (interquartile range) for continuous variables. We calculated $p$ for trend in each variable across the 3 categories using Cuzick's nonparametric test for trend [10]. We also described baseline PCS and MCS scores and their distribution of changes after 2 years across each category. We estimated the average changes in PCS and MCS scores in each age group, after adjusting for gender and dialysis vintage with a multilevel mixed-effect linear regression model. The data of KDQOL-SF score were collected yearly. We assumed the variations in the KDQOL-SF score over the study period for each patient. In other words, measurements were obtained at different time points and were accordingly nested within the individual patients. Repeated observations within individuals were considered at level 1 and level 2. Thus, the intercept was considered random effects in each model. Patients within one facility are more likely to have more similar outcomes than patients in different establishments. We used robust sandwich covariance estimators to account for the effects of facility clustering [9]. We performed all statistical analyses using STATA (version 14.2; Stata Corp., College Station, TX, USA) software. A two-sided $p$ value $<0.05$ was considered statistically significant.

\section{Results}

A total of 4,806 participants were included in J-DOPPS phases 4 and 5. After exclusion of participants aged younger than 60 years and those without KDQOL-SF scores after 2 years, 892 patients were eligible for the present study (Fig. 1). Baseline characteristics of the included and excluded patients are shown in online suppl. Table; see www.karger.com/doi/10.1159/000509309 for all online suppl. material.

Table 1 demonstrates patient characteristics at baseline, hemodialysis treatment, and laboratory characteristics overall and by age category. Compared with younger patients, older patients showed a shorter dialysis vintage, 


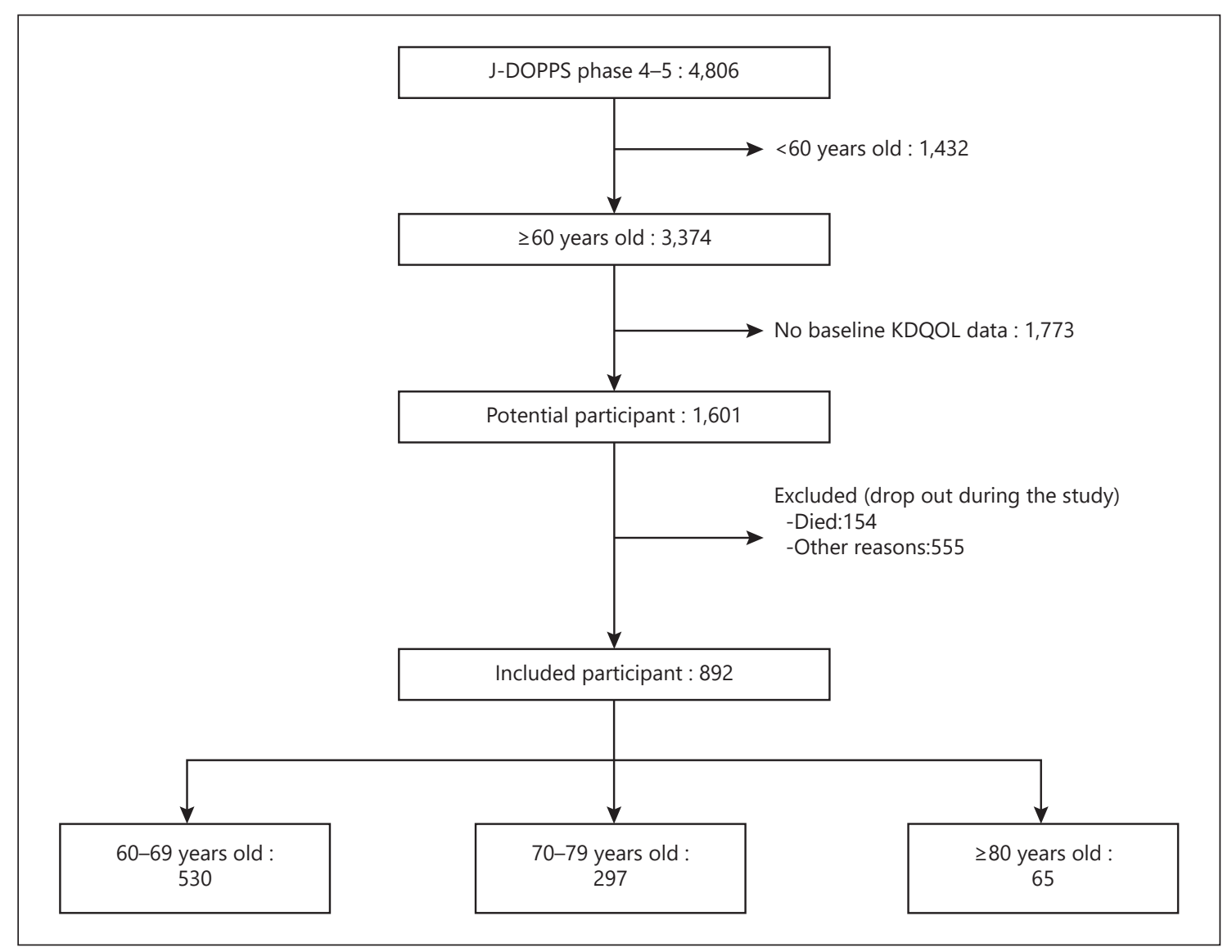

Fig. 1. Flowchart of the study.

higher proportion of those unable to walk, and higher prevalence of cancer other than skin, cerebrovascular disease, cardiovascular disease, hypertension, and mild cognitive impairment. There were no significant trends by type of vascular access, number of prescribed oral medications, and intradialytic hypotension across age categories. Serum creatinine and phosphate levels were lower in older patients than in younger patients.

Baseline KDQOL PCS and MCS scores overall and for the age categories are shown in Table 2. Hemodialysis patients aged $70-79$ and $\geq 80$ years had lower PCS scores than those aged 60-69 years (median: $70-79$ years $=43.1$; interquartile range $[\mathrm{IQR}], 35.2-49.4 ; \geq 80$ years $=38.8$; IQR, 31.6-43.8; 60-69 years $=45.4$; IQR, 37.5-51.4; $p<$ 0.001 ), while MCS scores did not significantly differ among age categories $(70-79$ years $=45.6$; IQR, 38.4$53.7 ; \geq 80$ years $=45.4 ;$ IQR, $36.9-55.1 ; 60-69$ years $=46.8$; IQR, 39.5-55.7; $p=0.1$ ).

Figure 2 shows the distribution of changes in PCS and MCS scores overall and by age groups. The MCS score decreased by 10 points or more in $25 \%$ of those aged 80 years and older. Tables 3-6 demonstrate changes in PCS and MCS scores with age category and patient characteristics. Those aged $70-79$ and $\geq 80$ years had significantly lower MCS scores than those aged 60-69 years, and males had significantly higher MCS scores than females, after 2 years. As dialysis vintage lengthened, the PCS score significantly decreased. In contrast, dialysis vintage was not associated with changes in the MCS score.

\section{Discussion/Conclusion}

This study shows that maintenance hemodialysis patients who were 70-79 and $\geq 80$ years old had lower PCS scores compared with those who were 60-69 years old, while MCS scores were not significantly different with age categories. As dialysis vintage lengthened, the PCS score significantly decreased, whereas dialysis vintage was not associated with the change in the MCS score. The MCS score 
Table 1. Participant and hemodialysis treatment characteristics by age category

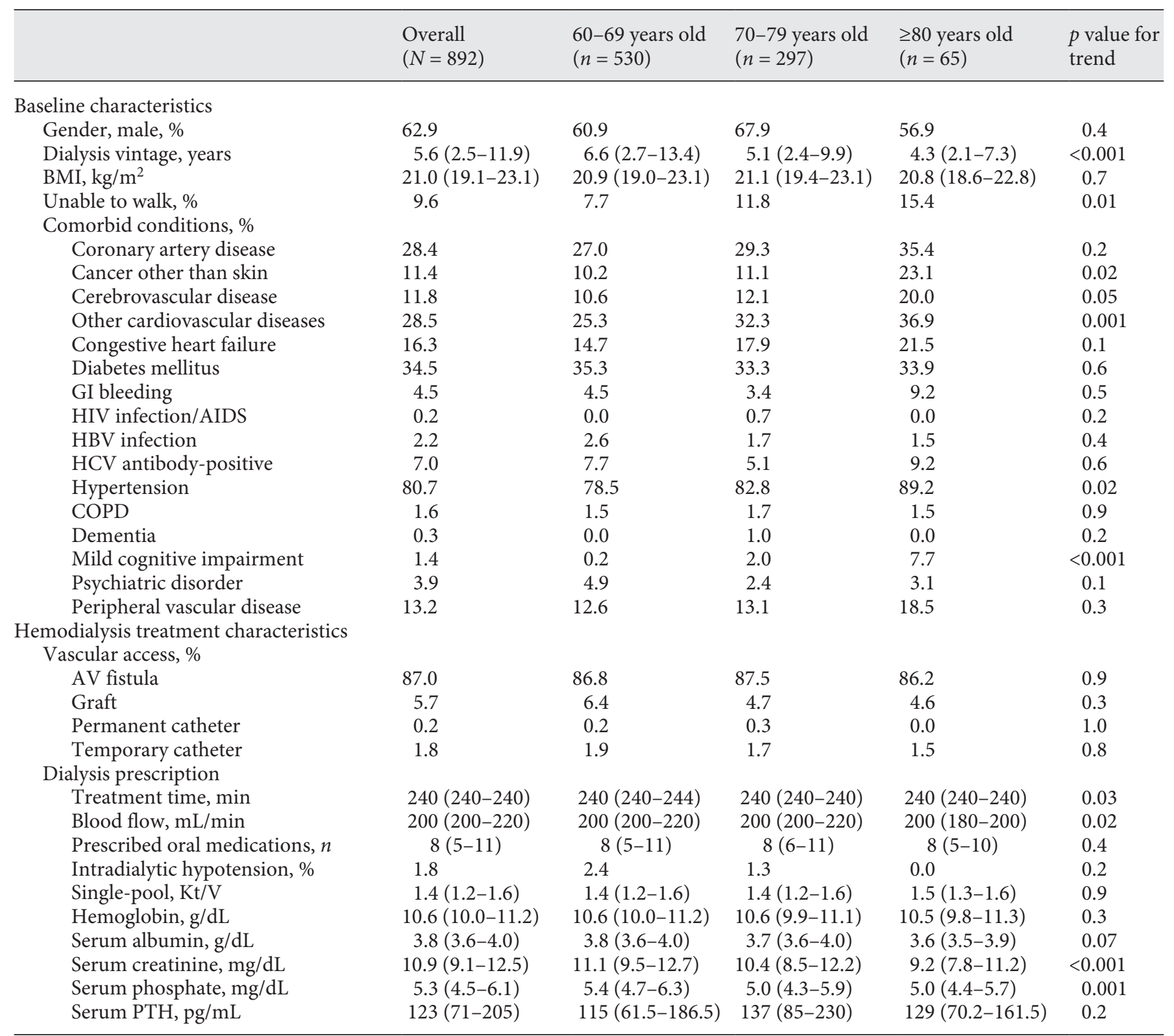

Data are presented as percentage or median (interquartile range). BMI, body mass index; GI, gastrointestinal; HIV, human immunodeficiency virus; AIDS, acquired immunodeficiency syndrome; HBV, hepatitis B virus; $\mathrm{HCV}$, hepatitis $\mathrm{C}$ virus; COPD, chronic obstructive pulmonary disease; AV, arteriovenous; PTH, parathyroid hormone.

Table 2. Baseline PCS and MCS scores

\begin{tabular}{llllll}
\hline & Overall & $60-69$ years old & $70-79$ years old & $\geq 80$ years old & $p$ value for trend \\
\hline PCS score & $44.0(36.8-50.2)$ & $45.4(37.5-51.4)$ & $43.1(35.2-49.4)$ & $38.8(31.6-43.8)$ & $<0.001$ \\
MCS score & $46.4(38.8-54.7)$ & $46.8(39.5-55.7)$ & $45.6(38.4-53.7)$ & $45.4(36.9-55.1)$ & 0.1 \\
\hline
\end{tabular}

Data are presented as median (interquartile range). PCS, physical component summary; MCS, mental component summary. 


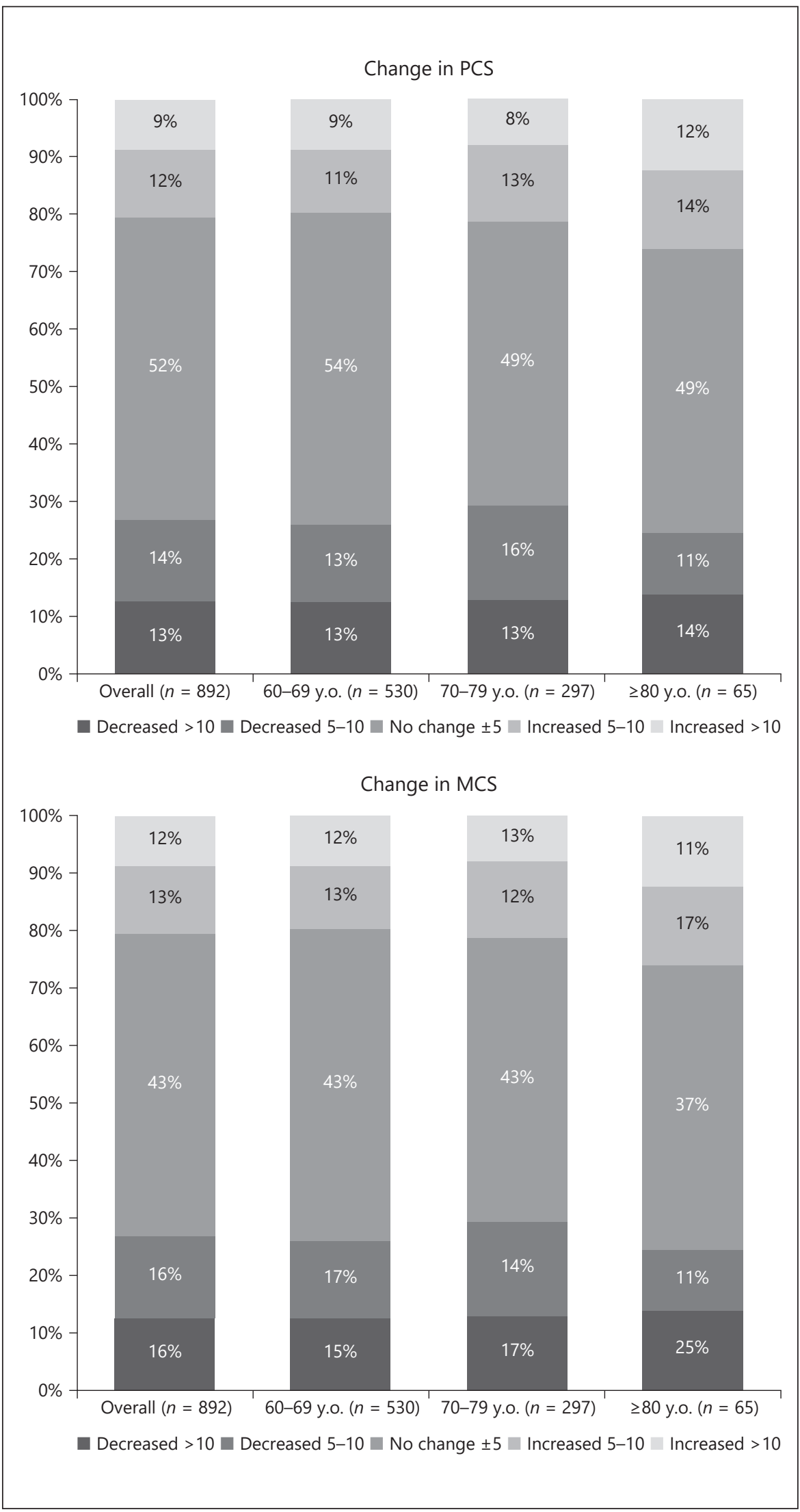

Fig. 2. Distribution of changes in PCS and MCS scores overall and by age group. PCS, physical component summary; MCS, mental component summary. 
Table 3. Change in the PCS score with age category

\begin{tabular}{llc}
\hline Parameter & Coefficient $(95 \% \mathrm{CI})$ & $p$ value \\
\hline Age category & & \\
$\quad 60-69$ y.o. & [Reference] & 0.02 \\
$\quad$ 70-79 y.o. & $-1.1(-2.0$ to -0.2$)$ & 0.09 \\
$\quad$ 280 y.o. & $-1.4(-3.0$ to 0.2$)$ & 0.003 \\
Years from study entry & $-0.8(-1.3$ to -0.3$)$ & $<0.001$ \\
Baseline PCS score & $0.6(0.6$ to 0.7$)$ & 0.7 \\
Gender, male & $0.2(-0.7$ to 1.0$)$ & 0.02 \\
Dialysis vintage, year & $-0.1(-0.1$ to -0.01$)$ & $<0.001$ \\
Intercept & $16.7(14.2$ to 19.2$)$ & \\
Intraclass correlation & $0.33(0.23$ to 0.46$)$ & \\
\end{tabular}

PCS, physical component summary; CI, confidence interval; y.o., years old.

Table 5. Change in the PCS score with age category (adjustment for comorbidities)

\begin{tabular}{llc}
\hline Parameter & Coefficient $(95 \%$ CI $)$ & $p$ value \\
\hline Age category & & \\
$\quad$ 60-69 y.o. & [Reference] & \\
$\quad$ 70-79 y.o. & $-1.1(-2.0$ to -0.3$)$ & 0.01 \\
$\quad$ Years y.o. & $-1.6(-3.3$ to 0.02$)$ & 0.053 \\
Baseline PCS score & $0.8(-1.3$ to -0.2$)$ & 0.004 \\
Gender, male & $0.4(-0.5$ to 0.7$)$ & $<0.001$ \\
Dialysis vintage, year & $-0.1(-0.1$ to -0.03$)$ & 0.4 \\
Coronary artery disease & $-0.6(-1.6$ to 0.4$)$ & 0.002 \\
Cancer other than skin & $0.1(-1.2$ to 1.4$)$ & 0.2 \\
Cerebrovascular disease & $-1.0(-2.2$ to 0.3$)$ & 0.9 \\
Other cardiovascular diseases & $-0.3(-1.2$ to 0.6$)$ & 0.5 \\
Congestive heart failure & $0.0(-1.1$ to 1.20$)$ & 1.0 \\
Diabetes mellitus & $-1.4(-2.4$ to -0.5$)$ & 0.004 \\
Hypertension & $0.8(-0.3$ to 1.9$)$ & 0.2 \\
COPD & $-2.4(-5.9$ to 1.0$)$ & 0.2 \\
Dementia & $-6.3(-9.6$ to -3.00$)$ & $<0.001$ \\
Mild cognitive impairment & $2.4(-0.8$ to 5.6$)$ & 0.1 \\
Psychiatric disorder & $1.6(-0.1$ to 3.3$)$ & 0.06 \\
Peripheral vascular disease & $-0.4(-1.7$ to 0.8$)$ & 0.5 \\
Intercept & $17.5(15.0$ to 20.7$)$ & $<0.001$ \\
Intraclass correlation & $0.32(0.21$ to 0.44$)$ & - \\
\hline
\end{tabular}

PCS, physical component summary; CI, confidence interval; y.o., years old; COPD, chronic obstructive pulmonary disease.

declined over time in older patients, particularly among those aged 80 years and older after 2 years' follow-up.

Among older J-DOPPS patients, the physical component was significantly more affected by age than the mental component. This result correlated well with the substantial decline with older age in the rate of patients who
Table 4. Change in the MCS score with age category

\begin{tabular}{llc}
\hline Parameter & Coefficient $(95 \%$ CI $)$ & $p$ value \\
\hline Age category & & \\
$\quad$ 60-69 y.o. & [Reference] & \\
$\quad$ 70-79 y.o. & $-1.1(-2.2$ to -0.1$)$ & 0.04 \\
$\quad$ Y80 y.o. & $-3.6(-6.0$ to -1.3$)$ & 0.002 \\
Years from study entry & $-0.5(-1.2$ to 0.1$)$ & 0.1 \\
Baseline MCS score & $0.5(0.5$ to 0.6$)$ & $<0.001$ \\
Gender, male & $1.8(0.7$ to 2.9$)$ & 0.001 \\
Dialysis vintage, year & $0.03(-0.04$ to 0.1$)$ & 0.4 \\
Intercept & $22.1(19.3$ to 25.0$)$ & $<0.001$ \\
Intraclass correlation & $0.38(0.29$ to 0.48$)$ & \\
\hline
\end{tabular}

CI, confidence interval; y.o., years old; MCS, mental component summary.

Table 6. Change in the MCS score with age category (adjustment for comorbidities)

\begin{tabular}{lcc}
\hline Parameter & Coefficient $(95 \%$ CI $)$ & p value \\
\hline Age category & & \\
$\quad$ 60-69 y.o. & [Reference] & \\
$\quad$ 70-79 y.o. & $-1.2(-2.3$ to -0.1$)$ & 0.03 \\
$\quad$ 80 y.o. & $-3.8(-6.0$ to -1.5$)$ & 0.001 \\
Years from study entry & $-0.5(-1.2$ to 0.1$)$ & 0.1 \\
Baseline MCS score & $0.5(0.4$ to 0.5$)$ & $<0.001$ \\
Gender, male & $2.0(0.9$ to 3.1$)$ & $<0.001$ \\
Dialysis vintage, year & $0.02(-0.1$ to 0.1$)$ & 0.7 \\
Coronary artery disease & $-0.9(-2.1$ to 0.3$)$ & 0.1 \\
Cancer other than skin & $-0.7(-2.3$ to 0.9$)$ & 0.4 \\
Cerebrovascular disease & $-0.4(-1.9$ to 1.1$)$ & 0.6 \\
Other cardiovascular diseases & $0.8(-0.3$ to 1.9$)$ & 0.1 \\
Congestive heart failure & $0.0(-1.5$ to 1.4$)$ & 0.9 \\
Diabetes mellitus & $-1.1(-2.2$ to 0.1$)$ & 0.06 \\
Hypertension & $1.0(-0.4$ to 2.3$)$ & 0.2 \\
COPD & $-1.4(-5.9$ to 3.1$)$ & 0.5 \\
Dementia & $-14.6(-23.4$ to -5.8$)$ & 0.001 \\
Mild cognitive impairment & $1.6(-3.9$ to 7.2$)$ & 0.6 \\
Psychiatric disorder & $-2.8(-5.0$ to -0.6$)$ & 0.01 \\
Peripheral vascular disease & $-1.6(-3.2$ to -0.1$)$ & 0.04 \\
Intercept & $23.1(19.7$ to 26.5$)$ & $<0.001$ \\
Intraclass correlation & $0.36(0.27$ to 0.47$)$ & - \\
\hline
\end{tabular}

CI, confidence interval; y.o., years old; COPD, chronic obstructive pulmonary disease; MCS, mental component summary.

could walk without assistance (Table 1). It has been reported that low muscle mass is associated with worse physical QOL domains [11]. These observations suggest that older patients are more restricted in their physical function than mental function, which is compatible with data of the worldwide DOPPS reported in 2011 [12]. 
In our present study, changes in physical and mental QOL among older dialysis patients were affected by hemodialysis vintage and age, respectively. Because the burden of symptoms in those on dialysis is high [13], QOL is often poor and satisfaction with life falls after dialysis initiation [14]. Perl et al. [7] previously reported slightly more variability in change in MCS than in PCS regardless of hemodialysis vintage. Functional dependencies among elderly people on hemodialysis increase with older age [15], which might be related to the decline in HR-QOL observed in our study.

HR-QOL of dialysis patients is lower than that of the general population or patients who undergo kidney transplantation $[16,17]$. A low HR-QOL in dialysis patients is also associated with decreased survival and more frequent hospitalization $[5,18,19]$. Mapes et al. reported that with each 10-point decrease in the HR-QOL score, mortality risk increased 25\% for the PCS and 13\% for the MCS. For hospitalization, risk increased $15 \%$ for the PCS and $6 \%$ for the MCS. The associations between HR-QOL and outcomes were reported to be stronger in Japan than in Euro-DOPPS or in the United States [5]. Measurement of HR-QOL is important because earlier recognition of low HR-QOL may allow healthcare providers to identify potentially vulnerable patients who are at an increased risk of death. In addition, independent of its strong association with death, HR-QOL itself is a main outcome measure in ESRD care. However, routine measurement of HR-QOL is not performed in most countries except in the USA, while the worst percentage among DOPPS countries of $11 \%$ is Japan [7].

Different approaches are needed for individual variations in characteristics in older people. Older people tend to present with multiple comorbidities, a higher risk of cognitive dysfunction [20], frailty, sarcopenia, and sensory impairment as well as functional and psychological dependence. Frail adults with ESRD are more likely to experience worsening HR-QOL [21]. Sarcopenia is associated with worse nutritional and clinical conditions and was a predictor of hospitalization and mortality in elderly patients on maintenance hemodialysis [11]. Impaired functional status is significantly associated with early death after starting dialysis especially among older patients [22]. The fact that PCS and MCS scores declined in one-quarter of hemodialysis patients aged older than 80 years in our present study indicates the need for social and mental support by both medical staff and families with the advance of age.

Intervention to improve HR-QOL in older hemodialysis patients is not necessarily a simple issue. Hemodialysis patients spend 9-15 h per week on dialysis and those with more active intradialytic activities are reported to have better mental and kidney disease-specific HR-QOL [23]. Furthermore, a large international study demonstrated that social support enhances physical QOL [24]. Improving individual QOL and the physical and mental health of older hemodialysis patients will eventually reduce a substantial and growing burden on society, exemplified by rapidly increasing medical care costs and number of healthcare providers.

Our study's strength is its prospective observational design and largest sample size to date to assess longitudinal measurements of HR-QOL among a representative cohort of hemodialysis patients in Japan, employing extensive case-mix adjustment of comorbidities, biochemistry, and treatment-related variables. However, our results should be interpreted with consideration to the following limitations. First, we were only able to assess HR-QOL once per year and were unable to assess the impact of any adverse events that may have caused HR-QOL to decline or increase. Second, our study population was restricted to patients who had an HR-QOL assessment at the beginning of follow-up and 2 years after. Patients who missed completion of even 1 patient questionnaire were thus ineligible. Third, we did not adjust HR-QOL for socioeconomic, occupational, or living status, all of which could influence HR-QOL. Fourth, the accuracy of selfreported QOL in patients with cognitive impairment may be questionable. It is reported that self-rated patient QOL tended to be more overestimated than caregiver-rated QOL [25]. QOL evaluation in cognitive impairment warrants careful interpretation. Fifth, this study includes patients within 1 year after hemodialysis initiation. It is possible that the magnitude of QOL change is large during the dialysis induction period, and this may have affected the results.

In conclusion, physical QOL was worse in the older age group, whereas mental QOL did not significantly differ among groups. Changes in physical and mental QOL among older maintenance dialysis patients were affected by hemodialysis vintage and age, respectively. Mental QOL declined over time within a relatively short period among older hemodialysis patients. Our study suggests that older hemodialysis patients are at high risk for HRQOL deterioration and the need for social and mental support for older hemodialysis patients by medical staff and families with the advance of age and hemodialysis vintage. Additional studies are needed to identify olderage-related factors specific to HR-QOL and to identify possible interventions to improve HR-QOL. 


\section{Acknowledgements}

We would like to thank the Arbor Research Collaborative for Health, Ann Arbor, MI, USA, for administering the J-DOPPS and to express our appreciation for the support of J-DOPPS by Kyowa Hakko Kirin Co., Ltd., without restriction on publication. The DOPPS.org website lists the full details. We also thank the study nurses, physicians, and medical directors for the time and attention they have devoted to our study.

\section{Statement of Ethics}

Study approval was obtained from the Central Institutional Review Board of Tokyo Women's Medical University (Approval Numbers: 1527, 1826, 2143, 2388, and 2388R). Additional study approval and written patient consent were obtained as required by national and local ethics committee regulations. The present study complied with the Declaration of Helsinki.

\section{Disclosure Statement}

S.F. has acted as a scientific advisor to Kyowa Hakko Kirin. T.H. has consulted for and received lecture fees from Kyowa Hakko Kirin. M.N. is a consultant for Akebia, Astellas, AstraZeneca, Kyowa Hakko Kirin, GlaxoSmithKline, Daiichi Sankyo, Taisho, Mitsubishi Tanabe, Chugai, Japan Tobacco, and Boehringer Ingelheim and has received research funding from Astellas, Ono, Kyowa Hakko Kirin, Daiichi Sankyo, Takeda, Mitsubishi Tanabe, Chugai, Torii, and Bayer. The other authors have nothing to declare.

\section{Funding Sources}

The DOPPS was supported by Amgen, Kyowa Hakko Kirin, AbbVie, Sanofi Renal, Baxter Healthcare, and Vifor Fresenius Medical Care Renal Pharma. Additional support for specific projects and countries was provided by Keryx Biopharmaceuticals, Merck Sharp \& Dohme, Proteon Therapeutics, Relypsa, and F. Hoffmann-La Roche; in Canada, specifically, support was provided by Amgen, BHC Medical, Janssen, Takeda, and the Kidney Foundation of Canada (for logistics support); in Germany, specifically, support was provided by Hexal, Deutsche Gesellschaft für Nephrologie, Shire, and the WiNe Institute; and support for the Peritoneal DOPPS in Japan was provided by the Japanese Society for Peritoneal Dialysis. All support was provided without restrictions on publication.

\section{Author Contributions}

A.I., S.Y., S.F., and T.H. designed the study; S.Y. and S.F. analyzed the data; S.Y. made the figures; A.I., T.H., S.W., and S.F. drafted and revised the paper; M.N. and T.H. organized the research; and all authors approved the final version of the manuscript.

\section{References}

1 Hamer RA, El Nahas AM. The burden of chronic kidney disease. BMJ. 2006 Mar 11; 332(7541):563-4.

2 Jager KJ, van Dijk PC, Dekker F, Stengel B, Simpson K, Briggs JD, et al. The epidemic of aging in renal replacement therapy: an update on elderly patients and their outcomes. Clin Nephrol. 2003 Nov;60(5):352-60.

3 Robinson BM, Zhang J, Morgenstern $\mathrm{H}$, Bradbury BD, Ng LJ, McCullough KP, et al. Worldwide, mortality risk is high soon after initiation of hemodialysis. Kidney Int. 2014 Jan;85(1):158-65.

4 Yazawa M, Kido R, Ohira S, Hasegawa T, Hanafusa N, Iseki K, et al. Early mortality was highly and strongly associated with functional status in incident Japanese hemodialysis patients: a cohort study of the large national dialysis registry. PLoS One. 2016 Jun 7;11(6): e0156951.

5 Mapes DL, Lopes AA, Satayathum S, McCullough KP, Goodkin DA, Locatelli F, et al. Health-related quality of life as a predictor of mortality and hospitalization: the Dialysis Outcomes and Practice Patterns Study (DOPPS). Kidney Int. 2003 Jul;64(1):33949.

6 National Kidney Foundation. K/DOQI clinical practice guidelines for chronic kidney disease: evaluation, classification, and stratifica- tion. Am J Kidney Dis. 2002 Feb;39(2 Suppl 1):S1-266.

7 Perl J, Karaboyas A, Morgenstern H, Sen A, Rayner HC, Vanholder RC, et al. Association between changes in quality of life and mortality in hemodialysis patients: results from the DOPPS. Nephrol Dial Transplant. 2017 Mar 1;32(3):521-7.

8 Lowrie EG, Curtin RB, LePain SD. Medical outcomes study short form-36: a consistent and powerful predictor of morbidity and mortality in dialysis patients. Am J Kidney Dis. 2003 Jun;41(6): 1286-92.

9 Pisoni RL, Gillespie BW, Dickinson D, Chen K, Kutner MH, Wolfe RA. The Dialysis Outcomes and Practice Patterns Study (DOPPS): design, data elements, and methodology. Am J Kidney Dis. 2004 Nov;44(5 Suppl 2):7-15.

10 Cuzick J. A Wilcoxon-type test for trend. Stat Med. 1985 Jan-Mar;4(1):87-90.

11 Giglio J, Kamimura MA, Lamarca F, Rodrigues J, Santin F, Avesani CM. Association of sarcopenia with nutritional parameters, quality of life, hospitalization, and mortality rates of elderly patients on hemodialysis. J Ren Nutr. 2018 May;28(3):197-207.

12 Canaud B, Tong L, Tentori F, Akiba T, Karaboyas A, Gillespie B, et al. Clinical practices and outcomes in elderly hemodialysis patients: results from the Dialysis Outcomes and Practice Patterns Study (DOPPS). Clin J Am Soc Nephrol. 2011 Jul;6(7):1651-62.

13 Murtagh FE, Addington-Hall JM, Edmonds PM, Donohoe P, Carey I, Jenkins K, et al. Symptoms in advanced renal disease: a crosssectional survey of symptom prevalence in stage 5 chronic kidney disease managed without dialysis. J Palliat Med. 2007 Dec;10(6): 1266-76.

14 Da Silva-Gane M, Wellsted D, Greenshields H, Norton S, Chandna SM, Farrington K. Quality of life and survival in patients with advanced kidney failure managed conservatively or by dialysis. Clin J Am Soc Nephrol. 2012 Dec;7(12):2002-9.

15 Cook WL, Jassal S. Functional dependencies among the elderly on hemodialysis. Kidney Int. 2008 Jun;73(11):1289-95.

16 Leim YS, Bosch JL, Arends LR, HeijenbrokKal MH, Hunink MG. Quality of life assessed with the Medical Outcomes Study Short Form 36-Item Health Survey of patients on renal replacement therapy: a systematic review and meta-analysis. Value Health. 2007 Sep-Oct; 10(5):390-7.

17 Álvares J, Cesar CC, de Assis Acurcio F, Andrade EI, Cherchiglia ML. Quality of life of patients in renal replacement therapy in Brazil: comparison of treatment modalities. Qual Life Res. 2012 Aug;21(6):983-91. 
18 Kalantar-Zadeh K, Kopple JD, Block G, Humphreys MH. Association among SF36 quality of life measures and nutrition, hospitalization, and mortality in hemodialysis. J Am Soc Nephrol. 2001 Dec;12(12):2797-806.

19 Lopes AA, Bragg-Gresham JL, Satayathum S, McCullough K, Pifer T, Goodkin DA, et al. Health-related quality of life and associated outcomes among hemodialysis patients of different ethnicities in the United States: the Dialysis Outcomes and Practice Patterns Study (DOPPS). Am J Kidney Dis. 2003 Mar; 41(3):605-15.
20 Kurella M, Chertow GM, Fried LF, Cummings SR, Harris T, Simonsick E, et al. Chronic kidney disease and cognitive impairment in the elderly: the health, aging, and body composition study. J Am Soc Nephrol. 2005 Jul; 16(7):2127-33.

21 McAdams-DeMarco MA, Ying H, Olorundare I, King EA, Desai N, Dagher N, et al. Frailty and health-related quality of life in end stage renal disease patients of all ages. J Frailty Aging. 2016;5(3):174-9.

22 Kurella Tamura M, Covinsky KE, Chertow GM, Yaffe K, Landefeld CS, McCulloch CE. Functional status of elderly adults before and after initiation of dialysis. N Engl J Med. 2009 Oct 15;361(16):1539-47.
23 Warsame F, Ying H, Haugen CE, Thomas AG, Crews DC, Shafi T, et al. Intradialytic activities and health-related quality of life among hemodialysis patients. Am J Nephrol. 2018;48(3):181-9.

24 Untas A, Thumma J, Rascle N, Rayner $\mathrm{H}$, Mapes D, Lopes AA, et al. The associations of social support and other psychosocial factors with mortality and quality of life in the dialysis outcomes and practice patterns study. Clin J Am Soc Nephrol. 2011 Jan;6(1):142-52.

25 Andrieu S, Coley N, Rolland Y, Cantet C, Arnaud C, Guyonnet S, et al. Assessing Alzheimer's disease patients' quality of life: discrepancies between patient and caregiver perspectives. Alzheimers Dement. 2016 Apr;12(4):427-37. 